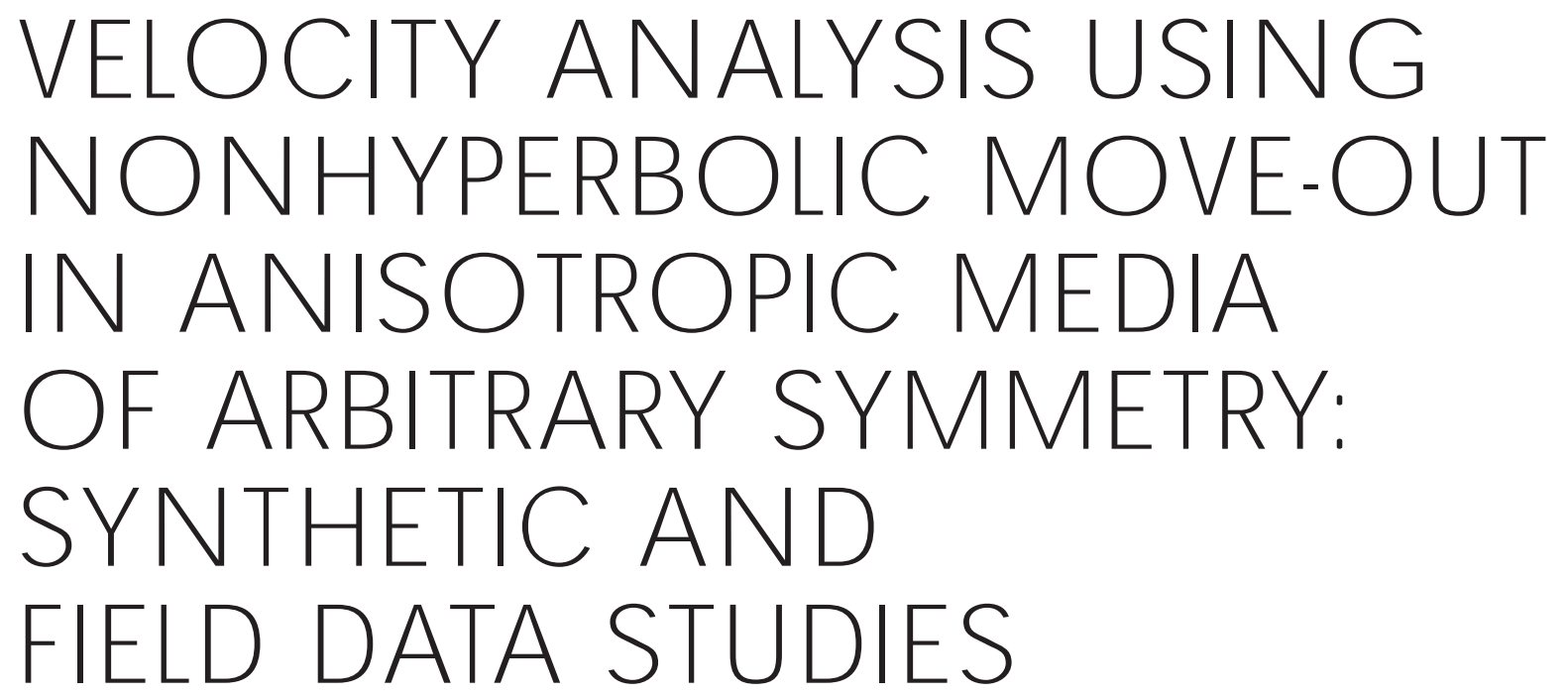

\author{
H. TABTI and P. RASO LOFOSAON \\ Institut français du pétrole ${ }^{1}$
}

\author{
ANALYSE DE VITESSE PAR CORRECTION \\ NON HYPERBOLIQUE DES INDICATRICES \\ DANS LES MILIEUX ANISOTROPES DE SYMÉTRIE \\ ARBITRAIRE : ÉTUDE SUR DES DONNÉES \\ SYNTHÉTIQUES ET RÉELLES.
}

Une méthode fiable, permettant d'estimer les paramètres d'intervalle (i.e. la vitesse de «normal move-out $V_{\text {NMO }}$ et le paramètre d'anisotropie $\mathrm{h}$ ) dans les milieux tabulaires transversalement isotropes à partir des ondes $\mathrm{P}$ réfléchies, a été proposée récemment par Alkhalifah (1997). Elle est basée sur l'équation du temps de trajet réfléchi développée par Tsvankin et Thomsen (1994). Cette méthode, testée sur des données synthétiques et expérimentales, consiste en une analyse de cohérence le long d'indicatrices non hyperboliques (i.e. comprenant les longs déports source-récepteur) permettant d'évaluer les paramètres effectifs suivis d'un processus de Dix pour l'estimation des paramètres d'intervalle. D'autre part, Sayers et Ebrom (1997) ont proposé de leur côté une autre expression non hyperbolique du temps de trajet ainsi que les expressions de Dix correspondantes valables pour les milieux tabulaires présentant des anisotropies azimutales. La méthode a été testée sur des modèles synthétiques homogènes présentant des anisotropies variées.

Nous proposons ici une généralisation de la méthode présentée par Alkhalifah, applicable à des milieux tabulaires d'anisotropie quelconque mais modérée (i.e. avec des degrés d'anisotropie pouvant aller jusqu'à environ $20 \%$ ). La paramétrisation de la vitesse est une extension naturelle de celle utilisée par l'auteur précédent. Elle s'exprime à l'aide des paramètres généralisés de Thomsen (1986) développés par Mensch et Rasolofosaon (1997).

Dans un premier temps, la méthode est appliquée sur des données synthétiques obtenues à partir d'un modèle à 6 couches présentant des anisotropies variées (à la fois le type et le degré). La robustesse de la méthode y est démontrée. En effet, tous les paramètres d'intervalle (ici $\mathrm{V}_{\mathrm{NMO}}$ et la vitesse horizontale $\mathrm{V}_{\mathrm{H}}$ ) sont estimés de façon satisfaisante (erreur $<2 \%$, à comparer aux degrés d'anisotropie qui varient de 15 à $20 \%$ ) et ce pour tous les azimuts. La méthode est ensuite appliquée sur des données réelles de mer du Nord comprenant trois profils sismiques se croisant au niveau d'un puits.
(1) 1 et 4 avenue de Bois-Préau, 92852 Rueil-Malmaison Cedex - France 


\section{VELOCITY ANALYSIS USING NONHYPERBOLIC MOVE-OUT IN ANISOTROPIC MEDIA OF ARBITRARY SYMMETRY: SYNTHETIC AND FIELD DATA STUDIES}

A robust method for estimating the interval parameters (i.e. the normal move-out velocity $\mathrm{V}_{\mathrm{NMO}}$ and the anisotropy parameter $\eta$ ) of horizontally layered transversely isotropic media from reflected P-waves data has been recently proposed by Alkhalifah (1997) based on move-out equation from Tsvankin and Thomsen (1994). The method, tested on synthetic and field data, is based first on semblance analysis on nonhyberbolic (i.e. long spread) move-out for the estimation of the effective parameters, and then on a layer stripping process. Sayers and Ebrom (1997) recently proposed another nonhyperbolic traveltime equation and a corresponding interval velocity analysis which can be used for azimuthally anisotropic layered media. The method was tested on synthetic and physical model data in homogeneous anisotropic media of various symmetry.

Here we propose a generalization of the method proposed by Alkhalifah, which can deal with arbitrary, but moderately (i.e. anisotropy strength of roughly $20 \%$ ), anisotropic layered media. The parametrization is a natural extension of the parametrization used by the previous author and based on generalized Thomsen's parameters (Thomsen, 1986) proposed by Mensch and Rasolofosaon (1997).

The method is first applied to synthetic data on a six layer model of contrasted anisotropy (type and magnitude). The robustness of the method is demonstrated. All the interval parameters (here $\mathrm{V}_{\mathrm{NMO}}$ and the horizontal velocity $\mathrm{V}_{\mathrm{H}}$ ) are estimated with reasonable errors (typically $<2 \%$, to be compared with the considered anisotropies of about 15 to $20 \%$ ) at all azimuths. The method is also tested on field data from the North Sea including three 2D seismic lines intersecting at a well location.

\section{ANÁLISIS DE VELOCIDAD UTILIZANDO MUDANZA NO HIPERBÓLICA EN MEDIOS ANISOTRÓPICOS DE SIMETRÍA ARBITRARIA: ESTUDIOS DE DATOS SINTÉTICOS Y DE TERRENO}

Un sólido método para estimar los parámetros del intervalo (es decir, la velocidad normal de mudanza $\mathrm{V}_{\mathrm{NMO}} \mathrm{y}$ el parámetro anisotrópico $\eta$ ) en medios transversalmente isotrópicos de capas horizontales a partir de datos de ondas $\mathrm{P}$ reflejas ha sido propuesto recientemente por Alkhalifah (1997) en base a la ecuación de desplazamiento de Tsvankin y Thomsen (1994). El método, estudiado en datos sintéticos y de terreno, se basa en primer lugar en el análisis de similitud de la mudanza no hiperbólica (es decir, de larga difusión) a partir de la estimación de los parámetros efectivos, y posteriormente en un proceso de remoción de capas. Sayers y Ebrom (1997) propusieron recientemente otra ecuación para el tiempo de recorrido no hiperbólico y un análisis correspondiente de velocidad del intervalo que puede utilizarse en medios estratificados azimutalmente anisotrópicos. El método fue probado sobre datos de modelos sintéticos y físicos de medios anisotrópicos homogéneos de simetría variada.
Proponemos aquí una generalización del método antes propuesto por Alkhalifah que puede tratar medios estratificados arbitraria pero moderadamente anisotrópicos (es decir, con potencia anisotrópica de aproximadamente $20 \%$ ). La parametrización es una extensión natural de la parametrización utilizada por el autor antes mencionado y se basa en parámetros de Thomsen generalizados (Thomsen 1986) propuestos por Mensch y Rasolofosaon (1997).

El método es aplicado en primer lugar a datos sintéticos de un modelo de seis capas con anisotropía contrastada (tipo y magnitud). Queda demostrada la solidez del método. Todos los parámetros del intervalo (aquí $\mathrm{V}_{\mathrm{NMO}}$ y la velocidad horizontal $\mathrm{V}_{\mathrm{H}}$ ) son estimados con errores razonables (error tipo $<2 \%$, comparado con las anisotropías consideradas, de 15 a $20 \%$ ) a todos los azimut. El método es probado también con datos de terreno del Mar del Norte, que incluyen tres líneas sísmicas bidimensionales que intersectan en la ubicación de un pozo. 


\section{INTRODUCTION}

Many field and laboratory measurements have proved the existence of elastic anisotropy in sedimentary rocks. These observations, together with the need of a better exploitation of seismic data records, make it necessary now to take into account the effects of anisotropy on seismic data analyses. The theoretical complexity of the description of the anisotropic wave propagation, due to the large number of elastic parameters required in anisotropic media, prevented the use of anisotropy in the conventional processing tools, despite some evidence of its existence (Helbig, 1994; Backus, 1962).

With the work of Thomsen (1986), under the weak anisotropy approximation in transversely isotropic media with a vertical axis of symmetry (TIV), a notable simplification to the problem was provided and a great step was passed towards the understanding of the influence of anisotropy on the seismic signatures of body waves. Since then many authors used this simplification to introduce transverse isotropy in the conventional processing tools, especially in the velocity analysis process (Byun et al., 1989; Sena, 1991; Tsvankin and Thomsen, 1994). Such an approach has been recently extended to TI media with non vertical symmetry axis and to orthorhombic media (Grechka and Tsvankin, 1996; Tsvankin, 1997). It remains, however, that the symmetry of geologic media is generally unknown. Furthermore, in the coordinate system associated to the seismic acquisition geometry, which is not necessarily aligned with the "crystallographic" axes of the prospected formations, the media may apparently be triclinic (Helbig, 1994). Thus, we address the following question: Is it possible to generalize the above approaches and deal with media of arbitrary symmetry type? The starting point is to provide a relevant parametrization of the medium. This was achieved by Mensch and Rasolofosaon (1997) which, under the weak anisotropy assumption, developed simple analytic expressions of the velocities of the three bulk waves by introducing a generalization of Thomsen's formulation to the general case of anisotropy. The coefficients of perturbation are dimensionless parameters which are the natural generalization of Thomsen's transversely isotropic parameters $\varepsilon, \delta$ and $\gamma$.

In this work, we use this velocity parametrization to develop a long spread (i.e. nonhyperbolic) move-out analysis (using semblance analysis) for reflected $q P$-waves valid in horizontally layered media of arbitrary symmetry. It will be shown, in particular, that in the weak anisotropy limit (a common case in practice), for each fixed azimuth the kinematic problem reduces to a TIV equivalent one. This is a very important result since it implies that most of the developments which were achieved for the TIV case can also be used in the most general case of anisotropy (i.e. triclinic) with an acceptable numerical accuracy. This fact is demonstrated here at least for the nonhyperbolic move-out analysis.

In the following, we first present a brief review of the $q P$-wave velocity parametrization developed by Mensch and Rasolofosaon (1997) in the general case of anisotropy. We then derive the time-distance equation and the generalized Dix formulation used in this work. After a description of the "anisotropic" semblance analysis process, we will show a test of the method on a synthetic model in order to demonstrate the robustness of the algorithm. Finally, we present the results of an application on real data from the North Sea.

\section{THEORETICAL BACKGROUND}

The main difficulty in the general case of anisotropy is the complexity of the velocity and the polarizations expressions since they include a large number of independent parameters. Yet, the weak anisotropy assumption allows us to overcome this difficulty as we will see below.

Consider a homogeneous anisotropic medium where space is referenced by a right handed coordinate system $x y z$ with the $z$-axis vertical, downwards (Fig. 1).

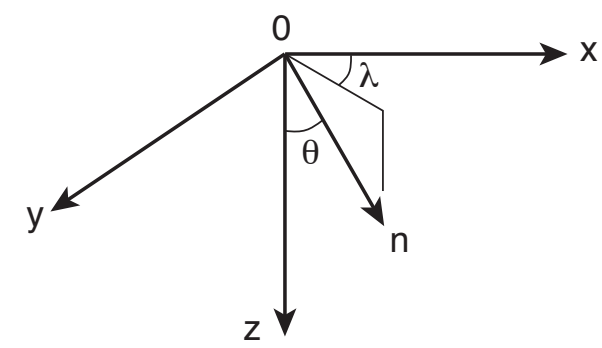

Figure 1

System of coordinate axes. $\boldsymbol{n}$ is the unit propagation vector referenced by the declination angle $\theta$ and the azimuthal angle $\lambda$. 
For a plane wave propagating in the direction $\boldsymbol{n}$, characterized by two polar angles (i.e. the declination $\theta$ and the azimuth $\lambda$ ), the velocity expression, in transversely isotropic media, is given, under the weak anisotropy assumption, by the first order equation (Thomsen, 1986):

$$
\frac{V_{P}(\theta, \lambda)}{V_{P 0}}=\frac{V_{\mathrm{P}}(\theta)}{V_{P 0}}=1+\delta S_{\theta}^{2} C_{\theta}^{2}+\varepsilon S_{\theta}^{4}
$$

$V_{P 0}$ is the vertical velocity, $S_{\theta}=\sin (\theta), C_{\theta}=\cos (\theta)$ and $\varepsilon$ and $\delta$ are the well known dimensionless anisotropy parameters which are combinations of the elastic stiffness coefficients of the TIV material.

Unlike the conventional exact formulation (that involves the $C_{I J}$ coefficients, see Auld (1973)), the previous linearized formulation (Eq. (1)) is particularly useful in understanding the behaviour of wave propagation in TIV media. This fact is well stressed by different authors (e.g. Tsvankin, 1996). For our purpose, we need to recall that the parameter $\varepsilon$ expresses the relative difference between the horizontal velocity and the vertical velocity, that is to say:

$$
\varepsilon \approx \frac{V_{P \text {-horizontal }}-V_{P 0}}{V_{P 0}}
$$

which is commonly called the " $P$-wave anisotropy". Note from Equation (1) that $\varepsilon$ controls the propagation at large angles $\theta$ (near horizontal directions).

The parameter $\delta$ has a simple physical interpretation in a reflection experiment. Consider a homogeneous TIV layer on which a reflection experiment is performed. If $X$ is the source-receiver distance and $T$ the traveltime of the reflected $q P$-wave, the initial slope of the $T^{2}-X^{2}$ curve is $1 / V_{N M O}^{2}$, where $V_{N M O}$ is the normal move-out $(N M O)$ velocity. In this context, the parameter $\delta$ represents the relative difference between the normal move-out velocity and the vertical velocity, i.e.

$$
\delta \approx \frac{V_{N M O}-V_{P 0}}{V_{P 0}}
$$

(Thomsen, 1986)

It is clear, from Equation (1) and from the above interpretation, that the parameter $\delta$ dominates the propagation at near vertical directions.

A natural continuation of the work of Thomsen was achieved by Mensch and Rasolofosaon (1997). In their work, these authors generalized the velocity parametrization to weakly anisotropic media of arbitrary symmetry (triclinic). With the geometry given in Figure 1, the $q P$-wave velocity in the direction $\boldsymbol{n}$, in media of arbitrary symmetry, now depends on both the declination $\theta$ and the azimuth $\lambda$ and is given by:

$$
\frac{V_{P}(\theta, \lambda)}{V_{P 0}}=1+\delta_{\lambda} S_{\theta}^{2} C_{\theta}^{2}+\varepsilon_{\lambda} S_{\theta}^{4}+\text { odd terms in } \theta
$$

where

$$
\delta_{\lambda}=\delta_{x} S_{\lambda}^{2}+\delta_{y} C_{\lambda}^{2}+2 \chi_{z} S_{\lambda} C_{\lambda}
$$

and

$$
\begin{aligned}
\varepsilon_{\lambda}=\varepsilon_{x} S_{\lambda}^{4} & +\varepsilon_{y} C_{\lambda}^{4}+\delta_{z} S_{\lambda}^{2} C_{\lambda}^{2} \\
& +2\left(\varepsilon_{16} C_{\lambda}^{2}+\varepsilon_{26} S_{\lambda}^{2}\right) S_{\lambda} C_{\lambda}
\end{aligned}
$$

The notations of Equation (2) are similar to those of Equation (1), that is to say $S_{\lambda}=\sin (\lambda)$ and $C_{\lambda}=\cos (\lambda)$. The odd terms in $\theta$ are not explicitly developed for reasons which will be discussed later. The weighting factors $\delta_{x}, \delta_{y}, \delta_{z}, \varepsilon_{x}$ and $\varepsilon_{y}$ appearing in Equations (2b) and (2c) are dimensionless anisotropic parameters which are the generalization to arbitrary symmetry of the Thomsen's TIV parameters $\delta$ and $\varepsilon$. As for the TIV case, they are simply a combination of the elastic stiffness coefficients of the medium (see Appendix). The subscripts $x, y$ and $z$ indicate that these parameters are relative to the plane normal to the $x$-, $y$ - and $z$-axis respectively. Furthermore, except for $\delta_{z}$, these parameters have exactly the same physical interpretation in the correponding coordinate planes as the Thomsen's TIV coefficients, i.e.:

$$
\begin{gathered}
\delta_{x} \approx \frac{V_{N M O}^{(y z-\text { plane })}-V_{P 0}}{V_{P 0}}, \delta_{y} \approx \frac{V_{N M O}^{(x z-\text { plane })}-V_{P 0}}{V_{P 0}}, \\
\varepsilon_{x} \approx \frac{V_{P \text {-horizontal }}^{(y z-\text { plane }}-V_{P 0}}{V_{P 0}}, \varepsilon_{y} \approx \frac{V_{P \text {-horizontal }}^{(x z-\text { plane }}-V_{P 0}}{V_{P 0}}
\end{gathered}
$$

(Mensch and Rasolofosaon, 1997), where $V_{N M O}^{(\pi-\text { plane) }}$ and $V_{P \text {-horizontal }}^{(\pi \text {-plane }}$ are respectively the $P$-wave $N M O$ velocity and the $P$-wave horizontal velocity in the plane $\pi(\pi=x z$ or $y z)$.

The remaining weighting factors $\chi_{z}, \varepsilon_{16}$ and $\varepsilon_{26}$ are new dimensionless anisotropic parameters which are also combinations of the elastic coefficients of the medium (see Appendix). They are closely related to 
eigendirections in the azimuthal variations of the anisotropic functions $\delta_{\lambda}$ and $\varepsilon_{\lambda}$ given in Equations (2b) and (2c) respectively. In this connection, as we will see below, the normal move-out velocity $V_{N M O}$ for a given azimuth $\lambda$ is given in the general case of anisotropy by:

$$
V_{N M O}(\lambda) \approx V_{P 0}\left(1+\delta_{\lambda}\right)(\text { Tabti et al., 1997) }
$$

We can show that the azimuthal variation of the normal move-out velocity is elliptical. This result is valid for arbitrary heterogeneous anisotropic media as demonstrated by Grechka and Tsvankin (1996). In a homogeneous medium the azimuth $\varphi$ of one of the axes of this ellipse is simply defined by:

$$
\tan (2 \varphi)=\frac{2 \chi_{z}}{\delta_{y}-\delta_{x}}
$$

(Rasolofosaon, 1997)

In TIV media we have:

$$
\chi_{z}=\varepsilon_{16}=\varepsilon_{26}=0, \varepsilon_{x}=\varepsilon_{y}=\varepsilon ; \delta_{z}=2 \varepsilon \text { and } \delta_{x}=\delta_{y}=\delta^{\prime}
$$

The parameter $\delta$ ' is equivalent to the Thomsen's parameter $\delta$ in the weak anisotropy limit, the difference being notable only for strong amounts of anisotropy (Sayers, 1994; Mensch and Rasolofosaon, 1997). Hence, Thomsen's Equation (1) can be considered as a particular case of the more general Equation (2).

More interesting is the form of the generalized Equation (2). It is clear that, if we except the odd terms in $\theta$, Equation (2) is formally identical to the TIV Equation (1) provided that $\delta$ is replaced by $\delta_{\lambda}$ and $\varepsilon$ by $\varepsilon_{\lambda}$.

The reason for which the odd terms in $\theta$ are not used here is that, for the geometry of propagation that interests us here (reflection surveys), these odd terms cancel each other along the symmetric raypath. More precisely, if we consider the raypath of a reflected $q P$-wave in horizontaly layered medium of arbitrary symmetry, we suppose that in each layer the up going ray always propagates with an opposite $\theta$ angle with respect to the downgoing ray. This is rigorously true for all the symmetry types down to monoclinic (with a horizontal symmetry plane). For the triclinic case we can show that for kinematic problems this assumption remains numerically accurate in the weak anisotropy limit since the deviation of the rays from the previous raypaths is of the second order in the perturbation (i.e. the anisotropic parameters).

Moreover, we can make an other simplification by considering that in horizontally layered media of

arbitrary but weak anisotropy, the overall raypaths in a reflection experiment are confined to the vertical plane containing the source and the receivers. Although this is not rigorously true except for TIV media, we can also show that the deviation of the rays from the vertical plane is of the second order in perturbation and thus negligible. This fact was largely verified on numerous models (Tabti et al., 1997) and was already emphasized by Chapman and Pratt (1992).

The previous statements lead to a very interesting conclusion. If we consider a reflection survey in a horizontally layered medium of arbitrary symmetry, for each azimuth $\lambda$, the kinematic problem can be treated as a TIV equivalent problem with $\delta=\delta_{\lambda}$ and $\varepsilon=\varepsilon_{\lambda}$. It is clear that in the general case of anisotropy the parameter $\varepsilon_{\lambda}$ represents, for each azimuth $\lambda$, the fractional difference between the horizontal velocity $V_{P \text {-horizontal }}(\lambda)$ and the vertical velocity in the vertical plane of azimuth $\lambda$, i.e:

$$
\varepsilon_{\lambda} \approx \frac{V_{P \text {-horizontal }}(\lambda)-V_{P 0}}{V_{P 0}}
$$

namely the $P$-wave anisotropy in that plane. Likewise, the parameter $\delta_{\lambda}$ represents the fractional difference between the normal move-out velocity $V_{N M O}(\lambda)$ and the vertical velocity in the vertical plane of azimuth $\lambda$, i.e.:

$$
\delta_{\lambda} \approx \frac{V_{N M O}(\lambda)-V_{P 0}}{V_{P 0}}
$$

It is straightforward from the above conclusion that any analytical development achieved in the case of horizontally layered TIV medium, that deals with $P$-waves reflection surveys and which uses Thomsen's formulation, can be directly applied to media of arbitrary symmetry only by replacing $\delta$ by $\delta_{\lambda}$ and $\varepsilon$ by $\varepsilon_{\lambda}$.

Hence, using the results in TIV media from Tsvankin and Thomsen (1994), Alkhalifah and Tsvankin (1995) and Alkhalifah (1997), the long spread time-distance curve of the reflected $q P$-wave in a horizontally layered medium of arbitrary symmetry is given, for a profile lying along an arbitrary azimuth $\lambda$ (Fig. 2), by:

$$
\begin{aligned}
T^{2}(X, \lambda) & =T_{0}^{2}+\frac{X^{2}}{V_{N M O}^{2}(\lambda)} \\
& -\frac{2 \eta(\lambda) X^{4}}{V_{N M O}^{2}(\lambda)\left\{T_{0}^{2} V_{N M O}^{2}(\lambda)+[1+2 \eta(\lambda)] X^{2}\right\}}
\end{aligned}
$$


where $T(X, \lambda)$ is the traveltime for the offset $X$ and for the azimuth $\lambda, T_{0}$ is the zero offset traveltime and $V_{N M O}(\lambda)$ is the average normal move-out velocity for azimuth $\lambda$. The parameter $\eta(\lambda)$, introduced in TIV media by Alkhalifah and Tsvankin (1995) and refined by Alkhalifah (1997), is the effective anisotropic parameter which is in our case azimuth dependent.

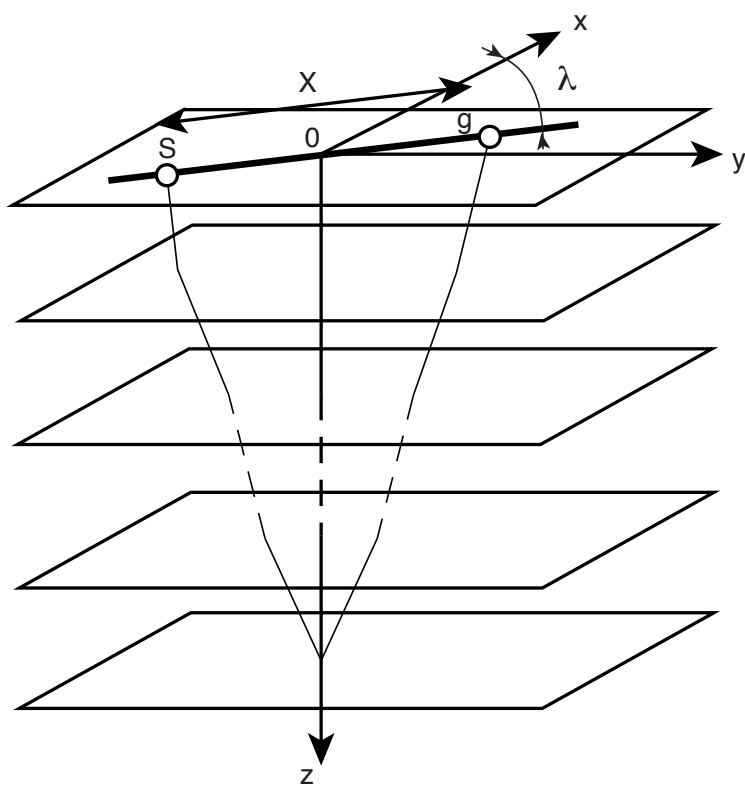

Figure 2

Reflection geometry in a weakly anisotropic horizontally layered medium. The raypath is contained in the vertical palne of azimuth $\lambda$.

The equations for the average quantities $V_{N M O}$ and $\eta(\lambda)$ in the general case of anisotropy are formally identical to the corresponding equations in TIV media (Alkhalifah, 1997) but are all azimuth dependent. The normal move-out velocity is given by:

$$
V_{N M O}^{2}(\lambda)=\frac{1}{T_{0}} \int_{0}^{T_{0}} v_{N M O}^{2}(\tau, \lambda) \mathrm{d} \tau
$$

where: $\quad v_{N M O}(\tau, \lambda)=V_{P 0}(\tau) \sqrt{1+2 \delta_{\lambda}(\tau)}$

is the interval normal moveout velocity in the vertical plane of azimuth $\lambda, V_{P 0}(\tau)$ is the interval vertical velocity and $\delta_{\lambda}(\tau)$ is the interval move-out parameter for azimuth $\lambda$ given by Equation ( $2 b$ ).
The effective anisotropic parameter is expressed as:

$$
\eta(\lambda)=\frac{1}{8}\left[\frac{1}{T_{0} V_{N M O}^{4}(\lambda)} \cdot \int_{0}^{T_{0}} v_{N M O}^{4}(\tau, \lambda)\left[1+8 \eta_{\lambda}(\tau)\right] d \tau-1\right]
$$

where:

$$
\eta_{\lambda}(\tau)=\frac{\varepsilon_{\lambda}(\tau)-\delta_{\lambda}(\tau)}{1+2 \delta_{\lambda}(\tau)}
$$

is the interval anisotropic parameter, $\varepsilon_{\lambda}(\tau)$ and $\delta_{\lambda}(\tau)$ being given by Equations (2b) and (2c). We introduce at this stage the average horizontal velocity corresponding to the azimuth $\lambda$. It is given by:

$$
V_{h}(\lambda)=V_{N M O}(\lambda) \sqrt{1+2 \eta(\lambda)}
$$

The very good fit, even at large offsets, between the predicted traveltime (Equations (3), (4) and (5)) and the corresponding exact traveltime was clearly demonstrated by Alkhalifah (1997) in TIV media. Our tests (not presented here) also show that, in weakly anisotropic (anisotropy strength of roughly 20\%) media of arbitrary symmetry, these equations predicts, at all azimuths, the exact traveltimes with the same very good fitness as in TIV media.

In order to compute the interval quantities appearing in the above equations, namely $V_{N M O}(\tau, \lambda)$ and $\eta_{\lambda}(\tau)$, from the average quantities (i.e. in the inverse problem) one can deduce the generalized Dix formulae by simple manipulations of Equations (4) and (5).

Furthermore, if the depths of the reflectors are known from additional experiments (VSP or logs) and provided that a sufficient number of azimuth profiles are available, one can compute the generalized anisotropic parameters appearing in Equations (2b) and (2c). The depth is necessary to first compute the left arguments of Equations (2b) and (2c). As pointed out by many authors, it is in fact impossible to estimate the depths of geologic interfaces only from reflections surveys in anisotropic media. Note from Equations (2b) and (2c) that at least 5 independent azimuths are necessary to make a full inversion of the anisotropic parameters in the general case of anisotropy. Only 3 independent azimuths are necessary to invert for orthorhombic media. Three is also the minimum number of independent azimuths necessary to estimate eigen 
directions of the normal move-out velocity which can be related to dominant vertical fractures for instance (Grechka and Tsvankin, 1996; Tabti et al., 1997). In orthorhombic media the eigen directions of the normal move-out velocity coincide with the eigen directions of the medium.

To close this section, it is worth noting that, in horizontally layered weakly anisotropic media of arbitrary symmetry, one can not distinguish between a triclinic layer and its equivalent monoclinic layer with a horizontal symmetry plane (i.e. obtained by vanishing all the odd terms in Equation (2a)) with only a reflection survey. However the important thing to bear in mind is that we are still able to investigate triclinic layers and determine some of their characteristics without loss of accuracy for other more symmetric layers situated below it (see inversion of complex synthetic model in Tabti et al., 1997).

\section{SEMBLANCE ANALYSIS USING NONHYPERBOLIC MOVE-OUT}

The advantage of Equation (3) is that, for a given azimuth $\lambda$, even in media of arbitrary symmetry only two parameters (for instance $V_{N M O}(\tau, \lambda)$ and $\eta_{\lambda}(\tau)$, in Equation (3)) are necessary to describe the long spread time-offset curves, provided that the zero offset time is given. On the other side, as demonstrated in the previous section, the kinematics as given by Equation (3) is "totally" decoupled azimuth by azimuth. As we will see below, this is a great simplification to the problem of velocity analysis when using the semblance approach in the inversion process.

Figure 3 illustrates the way the semblance analysis is conducted in the general case of anisotropy.

First of all, as suggested by Figure 3, we will use in our analysis the average horizontal velocity $V_{H}(\lambda)$ given by Equation (6) instead of the effective anisotropic parameter $\eta(\lambda)$ of Equation (5). This choice is more suitable for the numerical calculations as it allows us to deal with quantities of the same dimension and of the same order of magnitude.

Thus, in our case, for a given reflector and for each azimuth, the traveltime depends upon the offset $X$, the zero offset traveltime $T_{0}$ and the two average velocities $V_{N M O}$ and $V_{H}$. The offset $X$ is known and the zero offset traveltime can be determined prior to the anisotropic semblance analysis, for instance by doing an isotropic

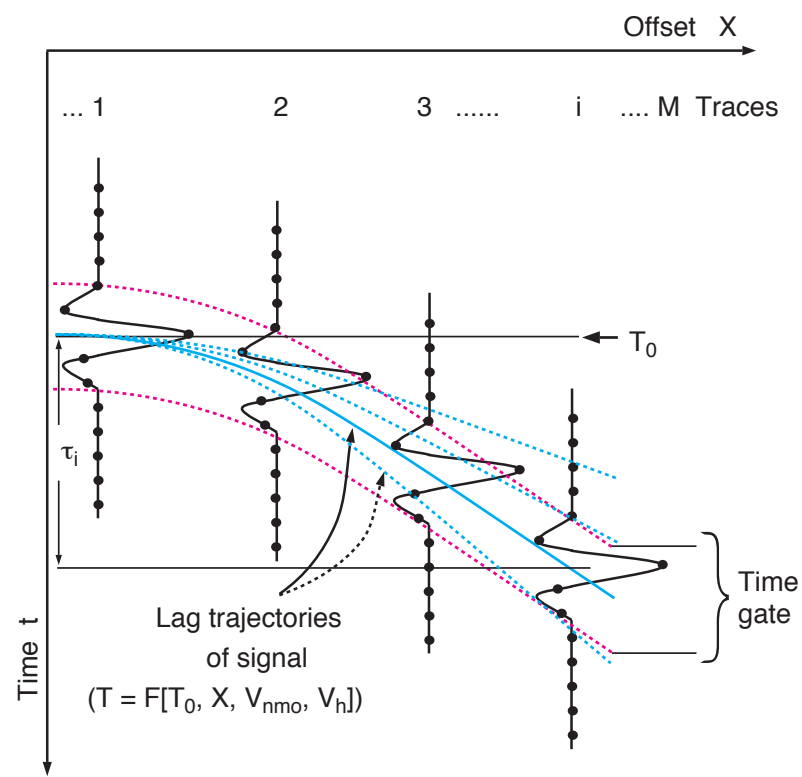

Figure 3

Anisotropic semblance analysis: measure of signal coherency along different time-lag trajectories caracterized by a fixed $T_{0}$ and different pairs of $\left(V_{N M O}, V_{h}\right)$. The cross correlations are evaluated over a "time gate" around each of the time-lag trajectories.

velocity analysis using only short offsets. This way of determining $T_{0}$ allows us at the same time to fix the velocity windows within which the two average velocities are scanned on during the semblance process. In fact, since the anisotropy is assumed to be weak, the widths of the scanning windows can be reduced around the value of the isotropic normal move-out velocity. Furthermore, this procedure leads to a significant reduction in the computation time. Naturally, the scanning windows can be extended in the case the anisotropy is stronger than expected.

Once $T_{0}$ is determined, $V_{N M O}$ and $V_{H}$ are scanned within the velocity windows and different time-offset curves are obtained (Fig. 3). Using the notations indicated in the Figure 3, the semblance function is computed for each couple of $V_{N M O}$ and $V_{H}$ (therefore for each of the time-lag trajectories (Fig. 3)) as follows (Taner and Koehler, 1969):

$$
S\left(\tau_{i}\right)=\frac{\sum_{t}\left[\sum_{i} f_{i}\left(t+\tau_{\mathrm{i}}\right)\right]^{2}}{M \sum_{t} \sum_{i} f_{i}^{2}\left(t+\tau_{i}\right)}
$$


where $f_{i}\left(t+\tau_{i}\right)$ is the sample value (amplitude) of the $i$ th trace at time $t+\tau_{i}, \tau_{i}$ is the move-out time for the $i$ th trace and $M$ the number of traces. The semblance function is a measure of the signal coherency among the different traces along a given time-lag trajectory. Thus, the maximum of the semblance function, which corresponds to the maximum of the reflected signal coherency, gives us the desired $V_{N M O}$ and $V_{H}$ velocities.

With the semblance analysis described above, the average (i.e. cumulative) normal move-out and horizontal velocities are estimated for each reflector and for each azimuth. Afterwards, using these average quantities and the zero offset traveltimes, the interval $N M O$ and horizontal velocities for each layer and for each azimuth can be computed using the generalized Dix formulae (deduced from Equations (4), (5) and (6)). Finally, the azimuthal variations of the interval velocities can be used to estimate symmetry directions in each layer.

\section{APPLICATION TO A SYNTHETIC MODEL}

The method described above is applied to synthetic data from a 6 layers model with a variety of symmetry directions (Fig. 4). The first layer is isotropic, the second is TI with a vertical axis of symmetry, the third layer is TI with an horizontal axis of symmetry parallel to the $x$ direction, the fourth layer is TI with an horizontal axis of symmetry lying at 60 degres from the $x$-axis, the fifth layer is isotropic, the sixth layer is TI with an horizontal axis of symmetry parallel to the $y$ axis and finally an isotropic layer which represents the basement. Since the coordinate system $x y z$ do not necessarily coincide with the "crystallographic" axes of each layer, the third, the fourth and the sixth layers appears respectively as orthorhombic, monoclinic and again orthorhombic. The generalized anisotropic parameters (see Appendix) and the vertical velocities of each layer are given in the Table 1.

The synthetic seimograms of the reflected $q P$-waves for 7 different azimuths were computed with the ray tracing code ANRAY developed by Gajewski and Psencik (1987). Figure 5 shows the synthetic seismogram for the azimuth $0^{\circ}$ (i.e. in the $x z$-plane)

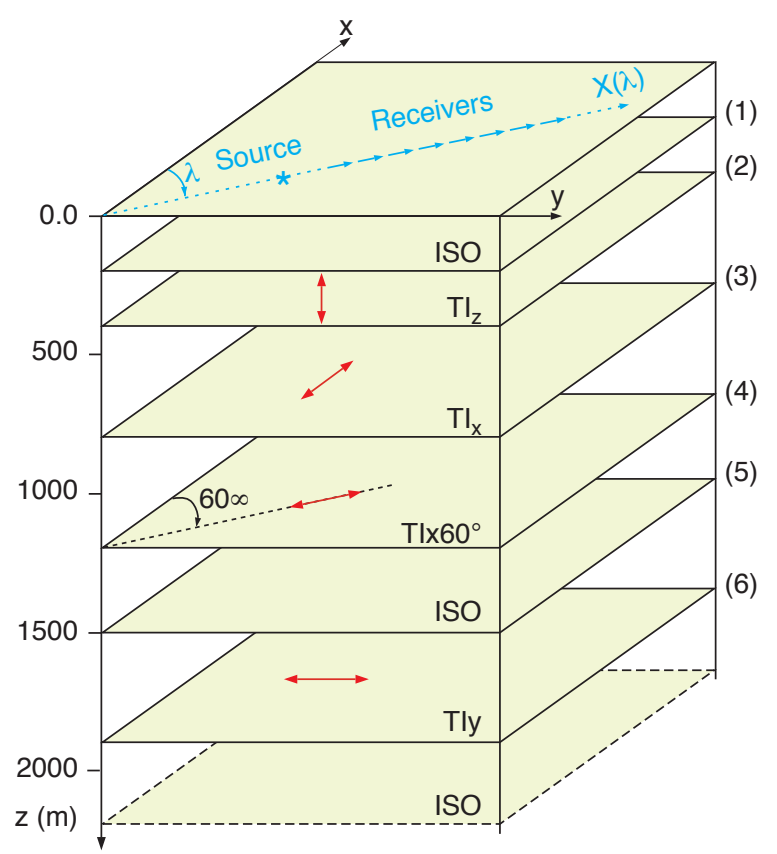

Figure 4

Six layers synthetic model. The layers are from top to bottom: (ISO) isotropic, (TIz) TI with a vertical axis of symmetry, (TI $x$ ) TI with a horizontal axis of symmetry parallel de the $x$-axis, (TI $60^{\circ}$ ) TI with horizontal axis of symmetry lying at $60^{\circ}$ from the $x$-axis, (ISO) isotropic, (TIy) TI with horizontal axis of symmetry parallel to the $y$-axis (ISO), isotropic basement. The model parameters are given in the table above.

TABLE 1

Model parameters

\begin{tabular}{c|c|c|c|c|c|c|c|c|c}
\hline Layer & $V_{P 0}(\mathrm{~km} / \mathrm{s})$ & $\varepsilon_{x}$ & $\varepsilon_{y}$ & $\delta_{x}$ & $\delta_{y}$ & $\delta_{z}$ & $\varepsilon_{16}$ & $\varepsilon_{26}$ & $\chi_{z}$ \\
\hline 1 & 1.5 & 0.0 & 0.0 & 0.0 & 0.0 & 0.0 & 0.0 & 0.0 & 0.0 \\
\hline 2 & 2.0 & 0.15 & 0.15 & 0.3 & 0.05 & 0.05 & 0.0 & 0.0 & 0.0 \\
\hline 3 & 2.8 & 0.0 & 0.1 & 0.0 & -0.09 & -0.09 & 0.0 & 0.0 & 0.0 \\
\hline 4 & 3.6 & 0.13 & 0.04 & 0.11 & 0.04 & 0.18 & 0.07 & 0.08 & 0.06 \\
\hline 5 & 3.2 & 0.0 & 0.0 & 0.0 & 0.0 & 0.0 & 0.0 & 0.0 & 0.0 \\
\hline 6 & 4.0 & 0.2 & 0.0 & 0.12 & 0.0 & 0.12 & 0.0 & 0.0 & 0.0 \\
\hline
\end{tabular}




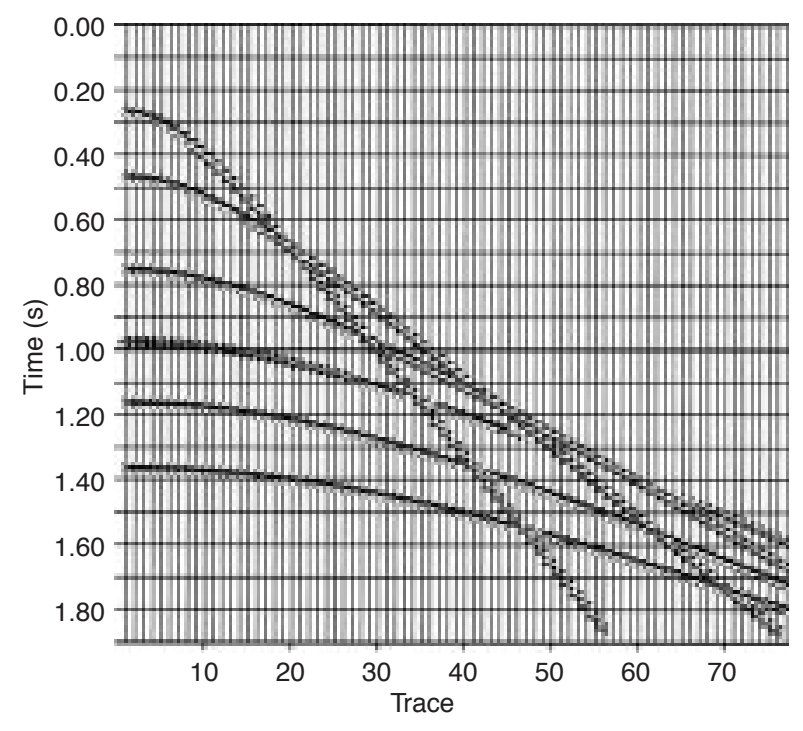

Figure 5

Synthetic seismogram from the previous model, corresponding to the azimuth $0^{\circ}$.

with the six reflection events. Figure 6 shows an example of the result of the semblance analysis on one of the six events for the previous azimuth. The desired $V_{N M O}$ and $V_{H}$ are obtained by picking the maximum of the semblance function as indicated on Figure 6.

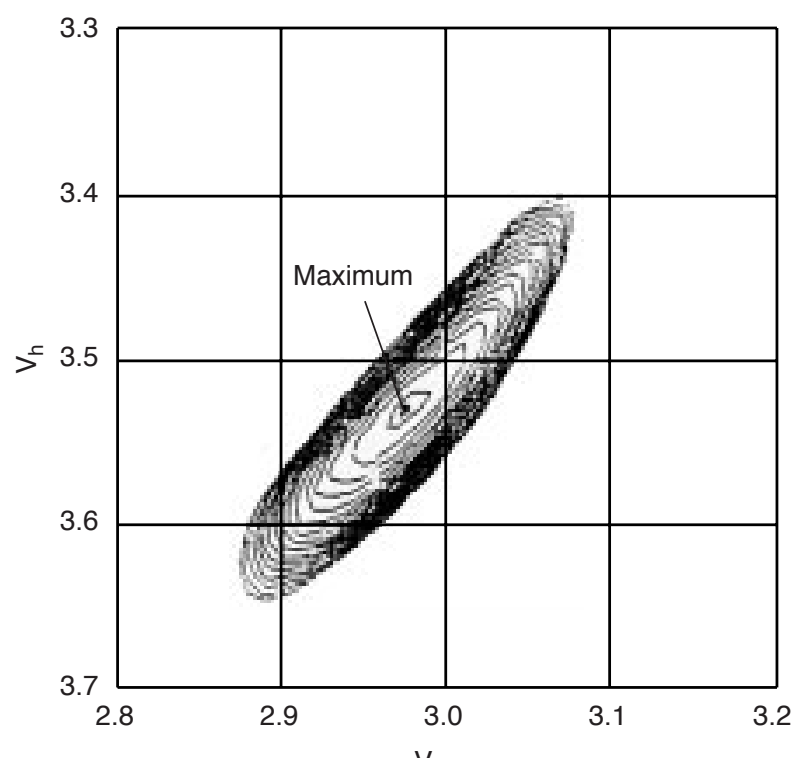

Figure 6

Semblance spectrum showing the maximum of the semblance function.
Here we will show only the results of the third and the sixth interfaces for the average velocities and those of the third and the sixth layers for the interval velocities. Although only these two results are presented, the conclusions are very similar for all the remaining layers. For all the events the average offset $X$ to depth $D$ ratio used is $\mathrm{X} / \mathrm{D} \approx 1.5$.

At first, the average velocities obtained by the semblance analysis are plotted on Figure 7 for the third interface and on Figure 8 for the sixth interface. In these two figures the exact velocities and the inverted velocities as function of the azimuth are in dashed and solid lines, respectively. The top part of these figures corresponds to the average normal move-out velocities and the bottom part to the average horizontal velocities.
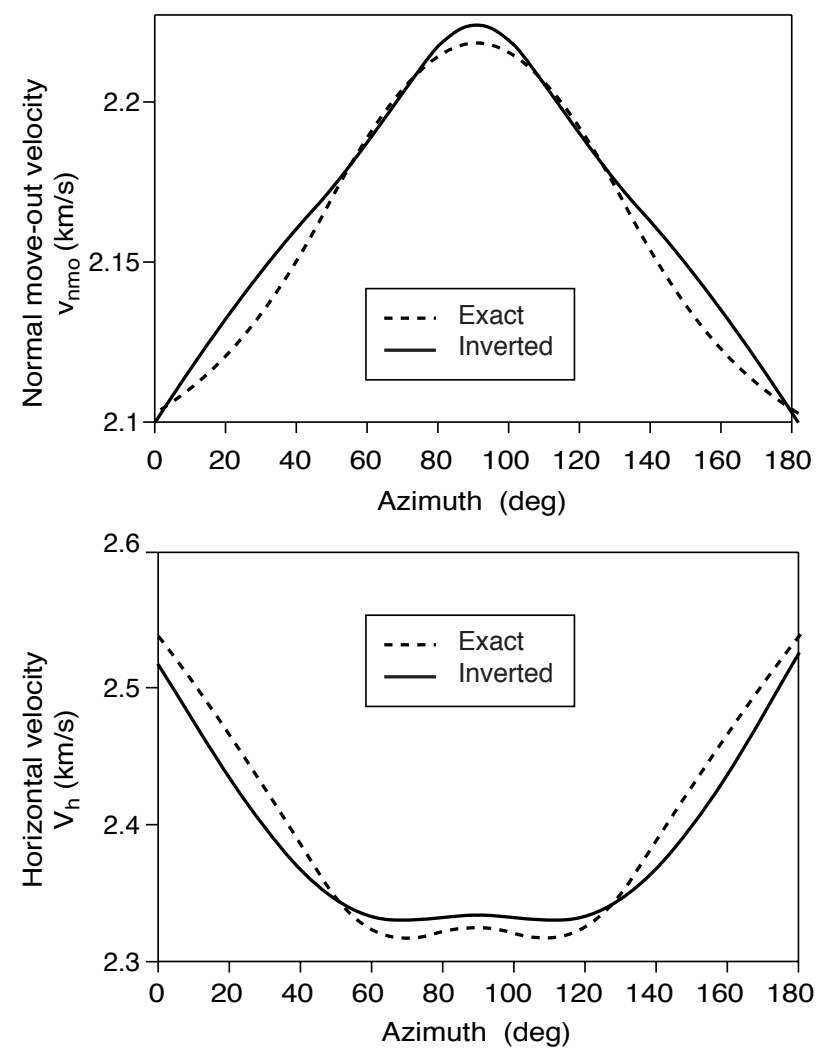

Figure 7

Exact and inverted average normal move-out velocities (top) and average horizontal velocities (bottom) as a function of azimuth at the third interface (i.e. bottom of the third layer). 
We can note the good fit between the exact velocities and the inverted velocities. The error made on the estimation of the average velocities is less than $1 \%$ for the normal move-out velocities and less than $2.5 \%$ on the horizontal velocities which can be considered as satisfactory as explained below. It has to be noted that the offset to depth ratio used limits the precision on the horizontal velocity inversion since this parameter controls essentially the far offsets propagation. Hence, the inversion of the horizontal velocity is generally less accurate than the normal move-out velocity.
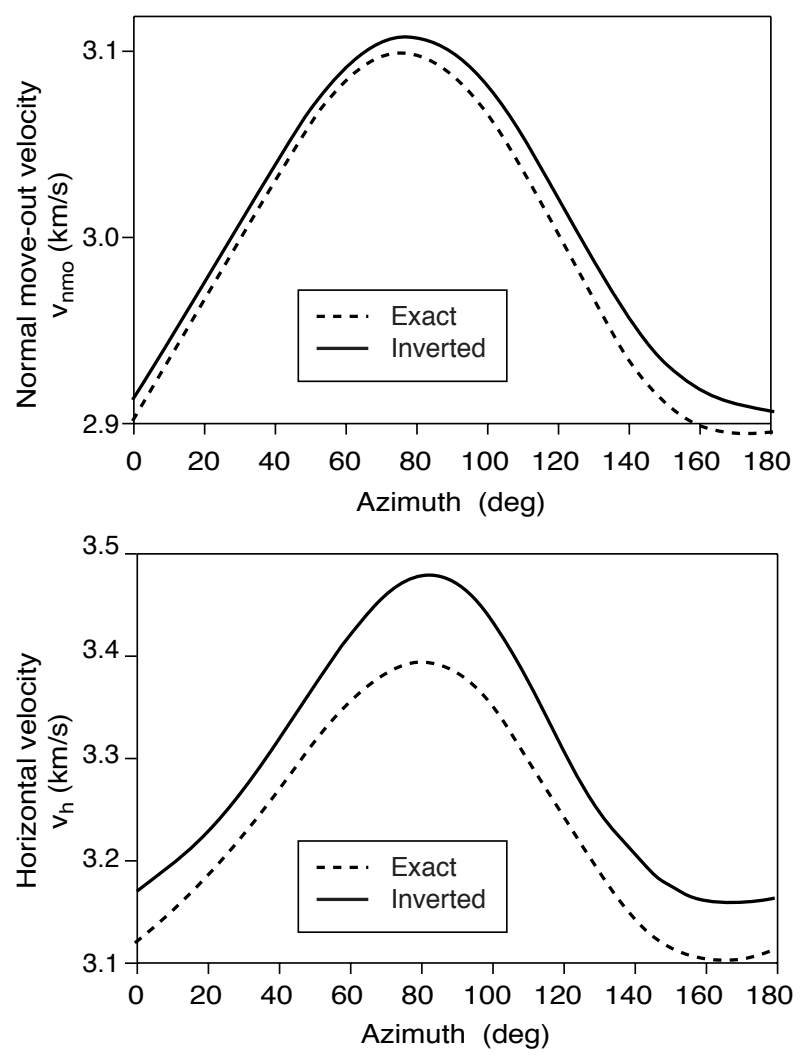

Figure 8

Exact and inverted average normal move-out velocities (top) and average horizontal velocities (bottom) as a function of azimuth at the sixth interface (i.e. bottom of the sixth layer).

The results of the interval velocities obtained from the generalized Dix formulae are presented on Figure 9 for the third layer and on Figure 10 for the sixth layer. As for the previous two figures, figures 9 and 10 show the exact velocities (in dashed lines) and the inverted velocities (in solid lines) as a function of azimuth. The top part of these Figures corresponds to the interval $N M O$ velocities and the bottom part to the interval horizontal velocities.
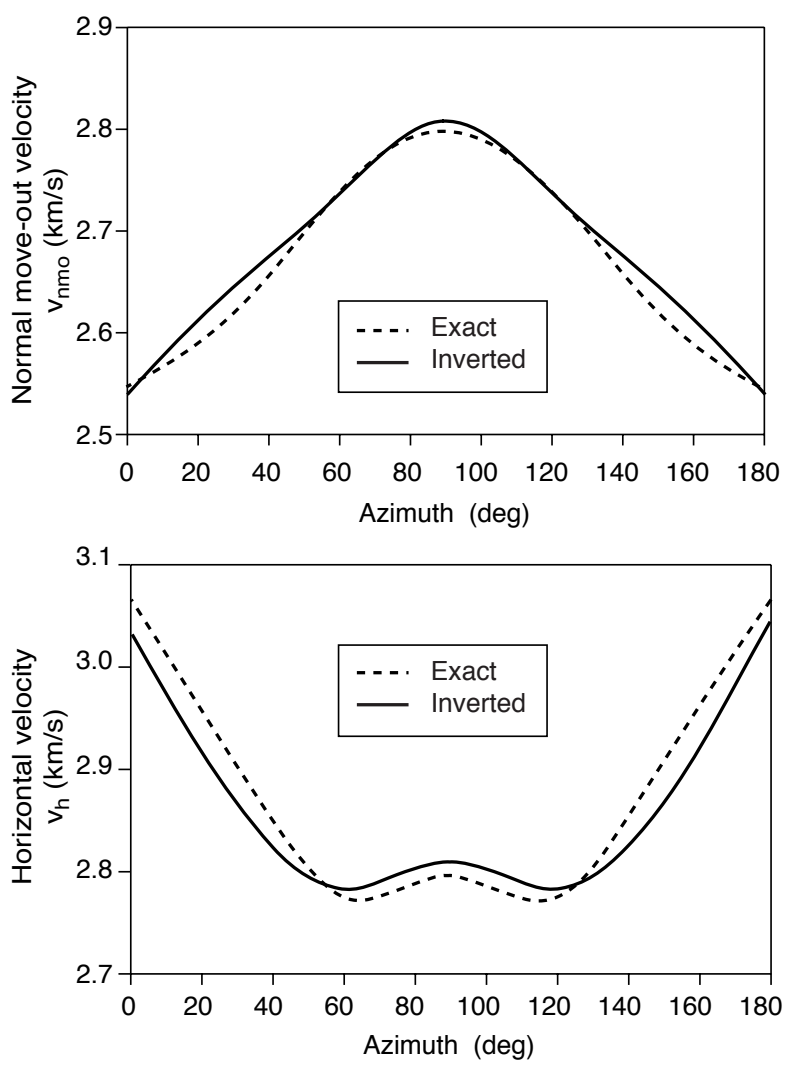

Figure 9

Exact and inverted interval normal move-out velocities (top) and interval horizontal velocities (bottom) as a function of azimuth in the third layer.

Again a good fit can be observed between the exact and the inverted velocities. The error made is less than $2 \%$ for the interval normal move-out velocities and less than $3 \%$ on the interval horizontal velocities which can be considered as satisfactory as explained a little further.

To give an idea on the amounts of anisotropy present in the model, the values of the interval parameters $\varepsilon_{\lambda}$ and $\delta_{\lambda}$ (defined by Equations (2b) and (2c)) for the two previous layers are plotted on Figures 11 (third layer) and 12 (sixth layer) as function of the azimuth $\lambda$. These amounts of anisotropy can also serve as a basis of comparison to the errors made on the inverted velocities. 

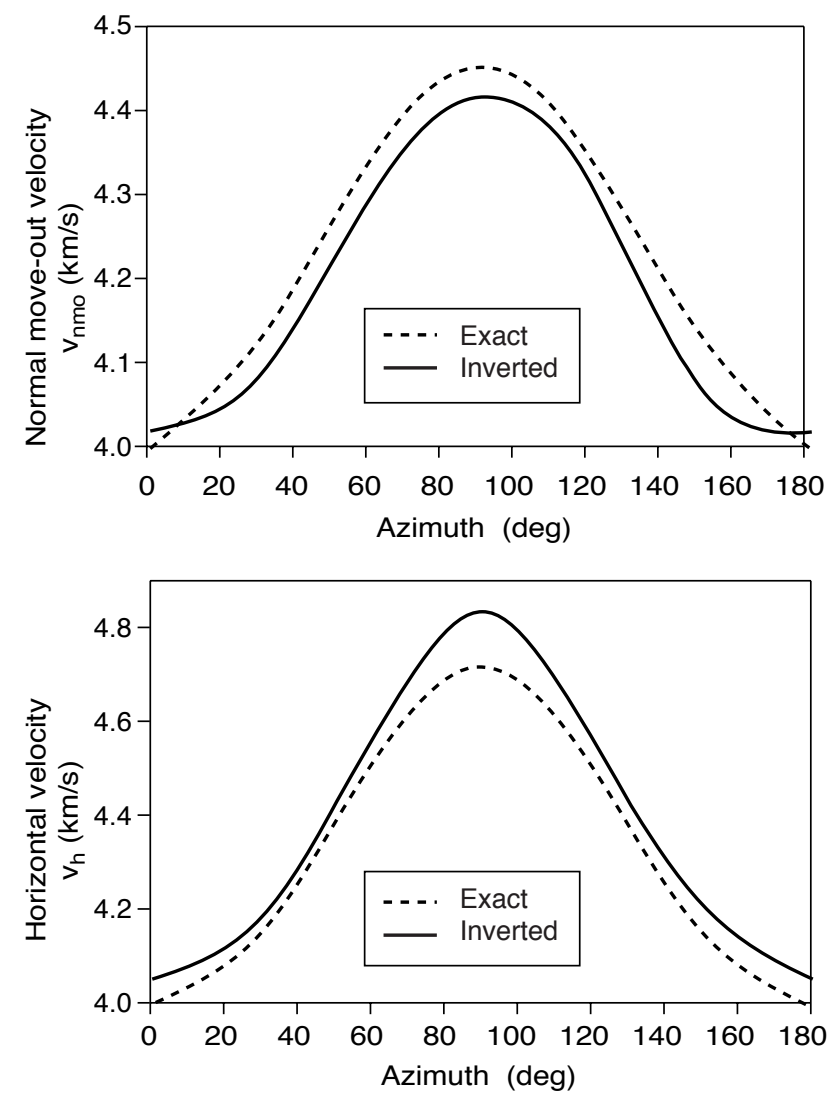

Figure 10

Exact and inverted interval normal moveout velocities (top) and interval horizontal velocities (bottom) as a function of azimuth in the sixth layer.
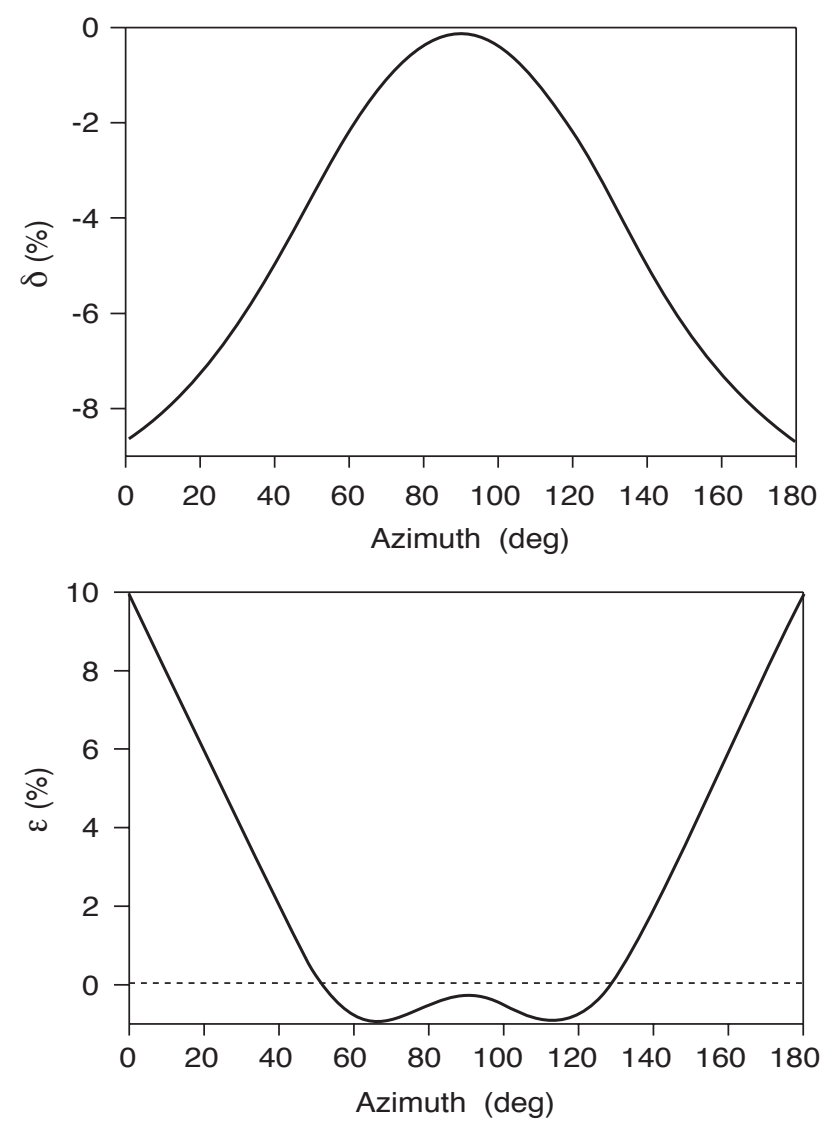

Figure 11

Interval parameters: $\delta_{\lambda}$ (top) and $\varepsilon_{\lambda}$ (bottom) as functions of azimuth $\lambda$ in the third layer.
Since $\varepsilon_{\lambda}$ and $\delta_{\lambda}$ quantify the relative difference between the horizontal velocity, respectively the $N M O$ velocity, and the vertical velocity, as previously mentioned, the azimuthal variations of the parameters $\varepsilon_{\lambda}$ and $\delta_{\lambda}$ evidently represents the $P$-wave azimuthal anisotropy of each layer which is of the order of $8 \%$ to $10 \%$ for the third layer and of $15 \%$ to $20 \%$ for the sixth layer. Thus we clearly see that the errors made on the inverted parameters, i.e. error on $N M O$ velocity $(<2 \%)$ and on horizontal velocity ( $<3 \%)$, are small compared to the variation of the parameters $\varepsilon_{\lambda}$ and $\delta_{\lambda}$, i.e. the azimuthal anisotropy. This is why we conclude that the inversion algorithm is satisfactory.

Finally, note that the gradients of the various parameters ploted in Figures 7-12 are not continuous at the wrap-around points (i.e. $0^{\circ}$ and $180^{\circ}$ ). This is due to the interpolation applied between the discrete points (i.e. the 7 azimuths).

\section{RESULTS ON REAL DATA}

In order to test its feasibility on real data, the method was also applied to three 2D surface seismic lines A, $\mathrm{B}$ and $\mathrm{C}$ intersecting at a well location (Fig. 13). Three CMPs around the well position are taken on each of the three lines. Seven seismic targets (horizons) were selected for the inversion process. Among the 7 inverted layers, the first layer is water.

A time-depth relation from the well data was used to estimate the depths of the reflectors and thus we could compute directly the interval parameters $\varepsilon_{\lambda}$ and $\delta_{\lambda}$ for each layer and for each of the 3 azimuths.

Figures 14 and 15 shows respectively one of the 3 CMPs used on line A (on which are reported the target horizons) and the corresponding semblance spectrum for the fourth event. The offset to depth ratio 

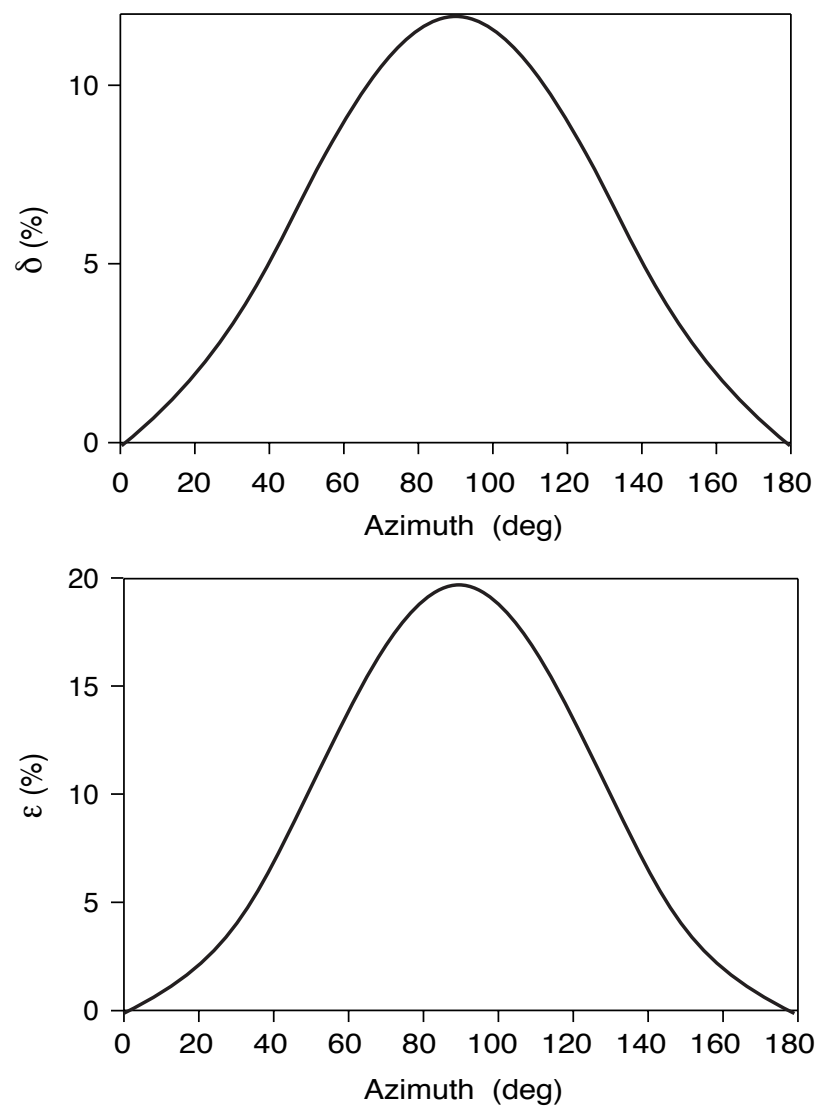

Figure 12

Interval parameters: $\delta_{\lambda}$ (top) and $\varepsilon_{\lambda}$ (bottom) as functions of azimuth $\lambda$ in the sixth layer.

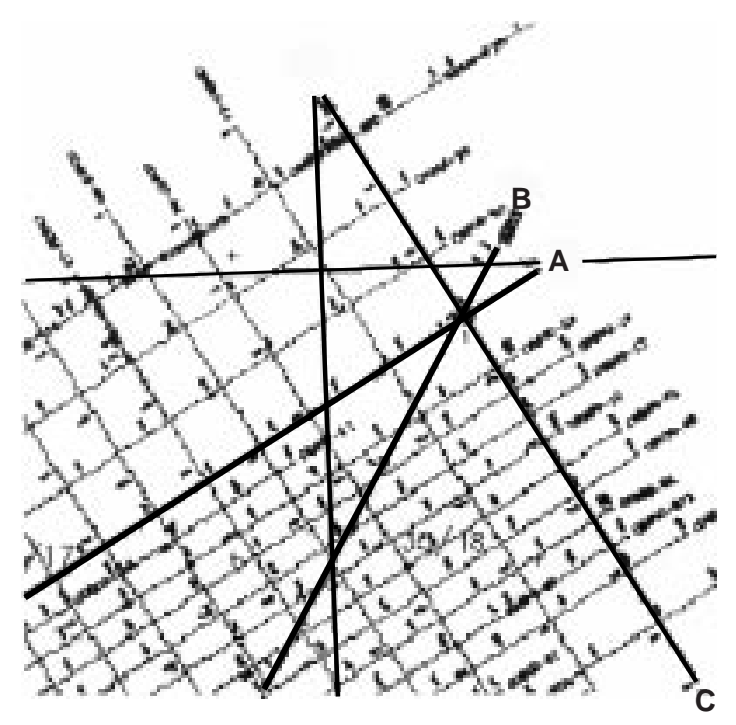

Figure 13

Field position of the three 2-D seismic lines A, B and C intersecting at a well location.

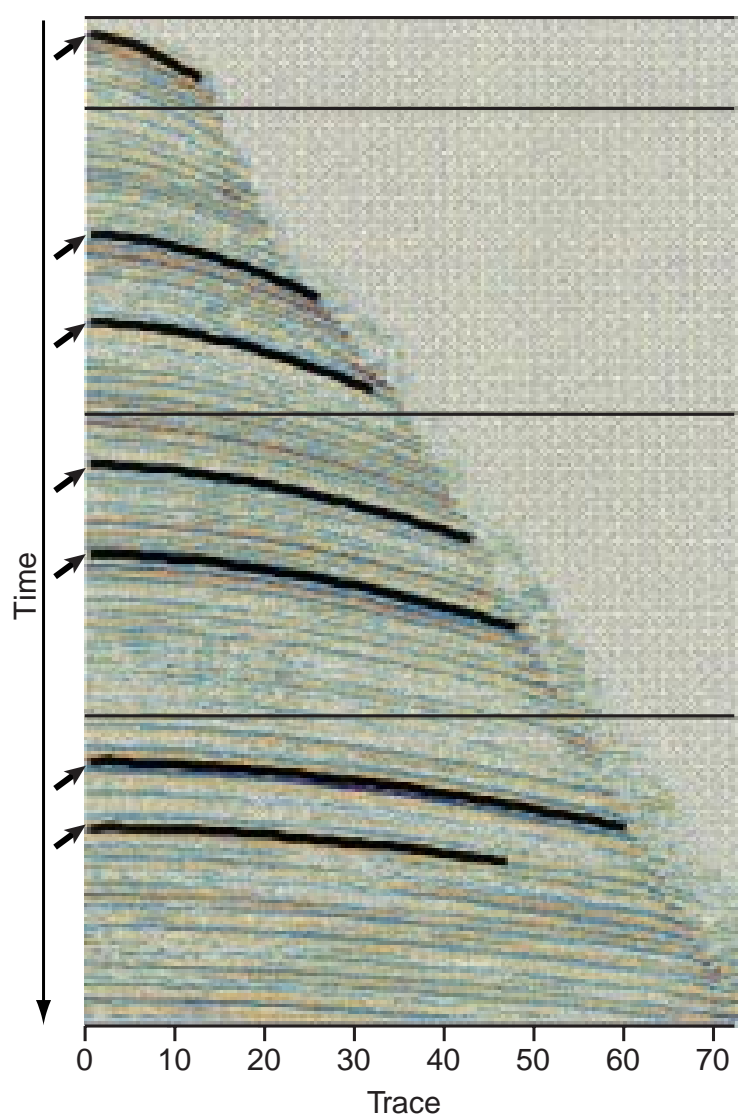

Figure 14

CMP gather used on line A. The target horizons are indicated by the arrows and the bold lines.

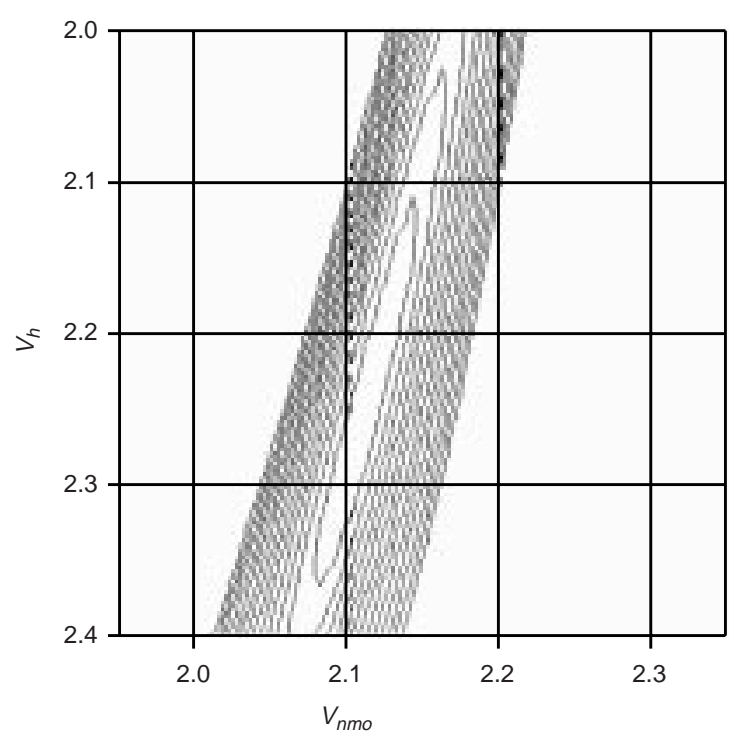

Figure 15

Semblance spectrum for the fourth event on line A (see Fig. 14). 
used for this event is roughly equal to 1 , which implies more uncertainties on the horizontal velocity as can be seen on Figure 15.

Note that, since we dispose only of 3 azimuths, the lowest symmetry level that we can consider is the orthorhombic symmetry. Without entering details, we shall note that, as the orientation of the horizontal orthorhombic axes are not a priori known, the method of inversion of the interval anisotropic parameters (i.e. the weighting factors in the right hand side of Equations (2b) and (2c)) consists in two steps. First the symmetry directions of the equivalent Orthorhombic layer are estimated from the values of the interval $N M O$ velocities in each layer. Basicaly this consists in fitting the azimuthal variation of the $N M O$ velocities to the best ellipse of which the principal axes are used as the new horizontal coordinate axes for the next step. Second the interval parameters of the equivalent orthorhombic medium are computed in the new coordinate system previously defined.

The results are summarized on the Figures 16 and 17. Figure 16 represents in color code the interval parameter $\delta_{\lambda}$ (in percent) for each of the 3 azimuths (i.e. the 3 lines A, B and C). On the right side of the figure are reported the symmetry directions of the equivalent orthorhombic layer and the maximum variations of the azimuthal anisotropy of the $P$-wave $N M O$ velocity in each layer.

Likewise, Figure 17 represents in color code the other interval parameter $\varepsilon_{\lambda}$ (in percent) for each of the three lines A, B and C.

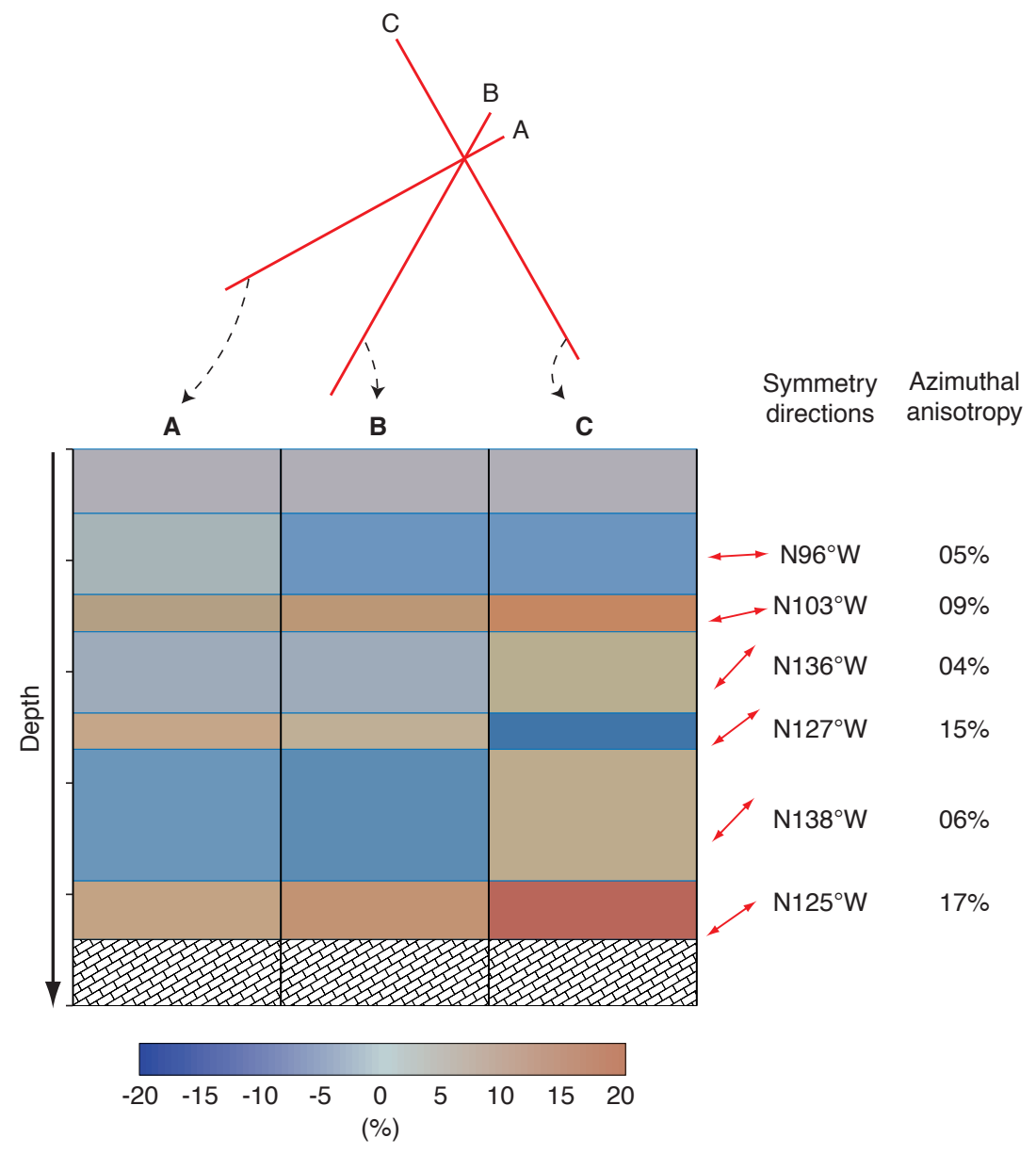

Figure 16

Results from the inversion of real data. Azimuthal dependence of the parameter $\delta(\lambda)$. On the right side are reported the symmetry directions and the retrieved maximum azimuthal anisotropy of the $N M O$ velocity. 
One can note from the two previous figures that almost all the layers exibits azimuthal variations of the parameters $\delta_{\lambda}$ and $\varepsilon_{\lambda}$ which indicates the existence of symmetries lower than TIV However, because of the presence of noise in real data, it is wiser to fix a lower limit beyond which one can really speak of azimuthal anisotropy. In our case, the second, the fourth and the sixth layers exibit only little variations of the parameter around zero $(+/-3 \%)$ which are of the same magnitude as the errors observed on synthetic data. It is reasonable to considere such observation as noise. Hence, only the third, the fifth and the seventh layer are considered to have an azimuthal anisotropy.

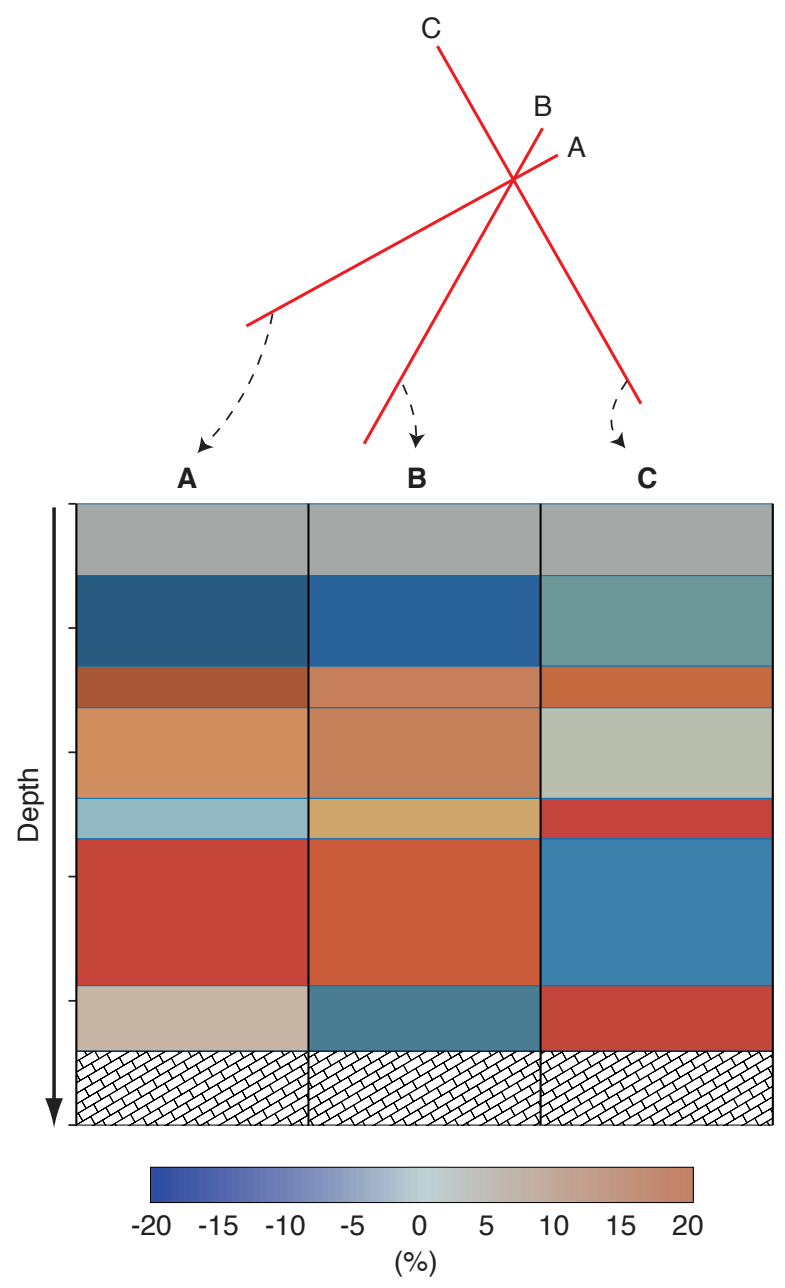

Figure 17

Results from the inversion of real data. Azimuthal dependence of the parameter $\varepsilon(\lambda)$
The fact that the orientation of the symmetry directions of these layers are not randomly oriented but clustered around an azimuthal direction of roughly $\mathrm{N} 105^{\circ} \mathrm{W}$ to $\mathrm{N} 125^{\circ} \mathrm{W}$ can be considered as a consistent result which probably has some geological explanation of which we are not aware.

We did not consider in the previous remark the parameters $\varepsilon_{\lambda}$ because we did not dispose in our inversion process of sufficient offsets to have confidence in the values obtained for this parameter. In fact, in the best case (i.e. the first layers) the offset was roughly equal to the depth of the reflector which is not enough to stabilise the inversion of this parameter.

Finally we mention that these results have not yet been compared with other information from well data because at the time of this draft, except the depths of the reflectors, such data was unavailable.

\section{DISCUSSION AND CONCLUSIONS}

Starting from the velocity parametrization in weakly anisotropic media of arbitrary symmetry developed by Mensch and Rasolofosaon (1997), we came up with a simple method for nonhyperbolic move-out analysis valid in media of arbitrary symmetry. We show that in the general case of anisotropy, the weak anisotropy assumption (a common case in the field, see Thomsen, 1986) allows us to decouple the kinematics azimuth by azimuth. As a consequence, the kinematic problem of reflected $q P$-waves in horizontally layered media is notably simplified since one can deal with arbitrary symmetries in any azimuth as if the layers were transversely isotropic with a vertical axis of symmetry. In other words, whatever is the symmetry type of the geologic layers in the investigated area, and whatever is the orientation of the seismic profile(s) in the field, it is always possible to make a nonhyperbolic normal moveout velocity analysis and to determine the interval parameters as in TIV media following the procedure described in recent publications (Alkhalifah, 1997). However, in the case we dispose only of one profile (one azimuth), the interval parameters must be considered as the $\varepsilon_{\lambda}$ and $\delta_{\lambda}$ (the generalized anisotropy parameters) for this particular azimuth.

The application of the method to a synthetic model with various symmetries demonstrated these facts. The analysis was conducted independently for each azimuth. Apart from the satisfactory errors made on the estimation 
of the average and the interval velocities, one can note that these errors are totally uncorrelated with the azimuthal variations of these velocities which confirms that the kinematics of the problem is decoupled azimuth by azimuth in the weak anisotropy limit.

Finally, the method was applied to real data to check its actual feasibility. Interval parameters and symmetry directions could be estimated and seem to lead to consistent results.

Weak anisotropy is the main assumption in this paper. A natural question arises: How weak is weak anisotropy for our particular problem? More precisely, up to which strength of anisotropy the proposed method remains robust?

The numerous numerical applications on contrasted models (i.e. symmetry types, anisotropy strength, etc.) have shown that the robustness of the proposed method is preserved for anisotropy strength up to $20 \%$.

\section{ACKNOWLEDGMENTS}

This work was partially supported by the Fonds de Soutien aux Hydrocarbures (COPREP) under the project G.2308/97. We wish to thank Total Exploration Production and the Institut français du pétrole (IFP) for the permission to publish these results. We are grateful to Tariq Alkhalifah of Stanford University, and to Paul Williamson of Elf Exploration Production for their careful review of this manuscript. We gratefully acknowledge Ivan Psencik (Geophysical Institute of Prague) and Dirk Gajewski (University of Hambourg) for allowing us to use their code ANRAY, and Laurence Nicoletis $(I F P)$ for fruitful discussions.

\section{REFERENCES}

Alkhalifah T. and Tsvankin I. (1995) Velocity analysis for transversely isotropic media. Geophysics, 60, 1550-1566.

Alkhalifah T. (1997) Velocity analysis using nonhyperbolic move-out in transversely isotropic media. Geophysics, 62, 18391854.
Auld B.A. (1973) Acoustic Fields and Waves in Solids, 1, Wiley, New York, NY.

Backus G.E. (1962) Long-wave elastic anisotropy produced by horizontal layering. J. Geophys. Res., 67, 4427-4440.

Byun B.S. Corrigan D. and Gaiser J.E. (1989) Anisotropic velocity analysis for lithology discrimination. Geophysics, 54, 1564-1574.

Chapman C.H. and Pratt R.G. (1992) Traveltime tomography in anisotropic media-1. Theory. Geophy. J. Int., 109, 1-9.

Gajewski D. and Psencik I. (1987) Computational of highfrequency seismic wave fields in 3-D laterally inhomogeneous anisotropic media. Geophys. J. R. Astr. Soc., 91, 383-411.

Grechka V. and Tsvankin I. (1996) 3-D description of normal move-out in anisotropic media. 66th Soc. Expl. Geophys. Annual Meeting, Expanded Abstracts, 1487-1490.

Helbig K. (1994) Foundations of anisotropy for exploration seismics. Handbooks of Geophysical Exploration, 22, Pergamon.

Mensch T. and Rasolofosaon P. (1997) Elastic-wave velocities in anisotropic media of arbitrary symmetry-Generalization of Thomsen's parameters $\varepsilon, \delta$ and $\gamma$. Geophys. J. Int., 128, 43-64.

Rasolofosaon P. (1997) Normal move-out from dipping reflectors in weakly anisotropic media of arbitrary symmetry. Submitted to Geophysics in March 1997.

Sayers C.M. (1994) P-wave propagation in weakly anisotropic media. Geophys. J. Int., 116, 799-805.

Sayers C.M. and Ebrom D.A. (1997) Seismic traveltime analysis for azimuthally anisotropic media: Theory and experiment. Geophysics, 62, 1570-1582.

Sena A.G. (1991) Seismic traveltime equations for azimuthally anisotropic media - Estimation of interval elastic properties. Geophysics, 56, 2090-2101.

Tabti H., Rasolofosaon P., Le Stunff Y. (1997) Generalized Dix formula and velocity analysis in weakly anisotropic media of arbitrary symmetry. 67th Soc. Expl. Geophys. Annual Meeting, Expanded Abstracts, 1242-1245.

Taner M.T., Koehler F. (1969) Velocity spectra-digital computer derivation and applications of velocity functions. Geophysics, 34, 859-881.

Thomsen L. (1986) Weak elastic anisotropy. Geophysics, 51, 1954-1966.

Tsvankin I. and Thomsen L. (1994) Nonhyperbolic reflection move-out in anisotropic media. Geophysics, 59, 1290-1304.

Tsvankin I. (1996) P-wave signatures and notation for transversely isotropic media: An overview. Geophysics, 61, 467483.

Tsvankin I. (1997) Move-out analysis for transversely isotropic media with a tilted symmetry axis. Geophysical Prospecting, 45, 479-512. 


\section{APPENDIX}

\section{Generalized anisotropy parameters}

The expressions of all the generalized anisotropy parameters used in this work and introduced by Mensch and Rasolofosaon (1997) are reported here for convenience.

$$
\begin{array}{ll}
\varepsilon_{x}=\frac{C_{22}-C_{33}}{2 C_{33}}, & \varepsilon_{y}=\frac{C_{11}-C_{33}}{2 C_{33}}, \\
\delta_{x}=\frac{C_{23}-C_{33}+2 C_{44}}{C_{33}}, & \delta_{\mathrm{y}}=\frac{C_{13}-C_{33}+2 C_{55}}{C_{33}}, \\
\delta_{z}=\frac{C_{12}-C_{33}+2 C_{66}}{C_{33}}, & \varepsilon_{26}=\frac{C_{26}}{C_{33}}, \\
\varepsilon_{16}=\frac{C_{16}}{C_{33}}, & \\
\chi_{z}=\frac{C_{36}+2 C_{45}}{C_{33}} &
\end{array}
$$

The coefficients $C_{I J}$ are the conventional elastic stiffness of the considered anisotropic medium expressed in Voigt notation with contracted indices (Helbig, 1994). 